\title{
Vector-like leptons: Higgs decays and collider phenomenology
}

\author{
A. Falkowski, ${ }^{a}$ D.M. Straub ${ }^{b}$ and A. Vicente ${ }^{c}$ \\ ${ }^{a}$ Laboratoire de Physique Théorique, CNRS - UMR 8627, Université de Paris-Sud 11, \\ F-91405 Orsay Cedex, France \\ ${ }^{b}$ Excellence Cluster Universe, Technische Universität München, \\ Boltzmannstr. 2, 85748 Garching, Germany \\ ${ }^{c}$ IFPA, Dep. AGO, Université de Liège, \\ Bat B5, Sart-Tilman B-4000 Liège 1, Belgium \\ E-mail: adam.falkowski@th.u-psud.fr, david.straub@tum.de, \\ avelino.vicente@ulg.ac. be
}

ABSTRACT: We study the impact of heavy vector-like leptons on several observables in collider and low-energy physics. These states, present in many well-motivated extensions of the Standard Model, can induce lepton flavour violation and non-standard Higgs decays. We study these effects in a model inspired by the composite Higgs scenario. After deriving bounds on the mass and production cross-section of the vector-like states using recent LHC data on multilepton searches, we discuss the modification of the Higgs decays to dilepton, diphoton and $Z \gamma$ final states as well as low-energy observables like radiative lepton decays, the anomalous magnetic moment of the muon and the electric dipole moment of the electron. We find several interesting correlations. In particular, we show that branching fractions of lepton flavour-violating Higgs decays at an observable level are prohibited due to the strong bounds on the radiative lepton decays.

KeYwords: Higgs Physics, Beyond Standard Model, Electromagnetic Processes and Properties

ARXIV EPRINT: 1312.5329 


\section{Contents}

1 Introduction 1

2 The model 2

3 Current experimental situation and future projects 4

3.1 Bounds on lepton flavour violation 4

$\begin{array}{lll}3.2 & \text { Bounds on vector-like leptons } & 6\end{array}$

4 Higgs decays and lepton flavour violation $\quad 7$

$\begin{array}{ll}4.1 \text { Higgs decays } & 7\end{array}$

$\begin{array}{llr}4.2 & \text { Low-energy observables } & 9\end{array}$

$\begin{array}{lll}4.3 & \text { Numerical analysis } & 11\end{array}$

$\begin{array}{lll}5 & \text { Summary and conclusions } & 13\end{array}$

\section{Introduction}

Since the start up of the Large Hadron Collider (LHC) in 2008, the performance of the collider and the results already achieved have been outstanding. The data collected by the ATLAS and CMS collaborations have provided conclusive evidence for the existence of the Higgs boson and put very stringent constraints in many new physics (NP) scenarios. Well-motivated frameworks, like supersymmetry or extra dimensions, have been searched with dedicated efforts, but so far no sign of them has been found.

The discovery of a Higgs boson $[1,2]$ with $m_{h} \approx 126 \mathrm{GeV}$ not only gave us the final missing piece in the Standard Model (SM), but also opened a window to search for new physics. So far all data seem to be consistent with the $126 \mathrm{GeV}$ particle being the SM Higgs boson (see e.g. [3-6]), but the possibility of deviations from the expected SM properties still remains and this has encouraged many studies in this direction. For example, new fermions with masses near the weak scale may alter the Higgs properties. Although a chiral 4th generation has already been excluded by the LHC (precisely due to the nonobservation of an enhancement in the Higgs production cross-section), the existence of one or more vector-like families [7] is a perfectly valid possibility. The contribution of these new states to Higgs radiative decays can lead to observable effects at the LHC.

In this paper we concentrate on vector-like leptons. More precisely, we are interested in models in which the SM charged leptons get their masses due to their mixing with massive vector-like states. This scenario allows for a simple understanding of the mass hierarchy among different generations and, in fact, it is common to many well-motivated extensions of the SM, such as composite Higgs models [8,9] and warped extra dimensions [10, 11]. 
We explore the phenomenology of vector-like leptons on several fronts. We will do so by means of a simple model that shares many aspects of the phenomenology of a wide class of models, such as composite Higgs models or warped extra dimensions. First of all, we need to determine the allowed parameter space when LHC data is taken into account. Once pair-produced, mostly in Drell-Yan processes [12], vector-like leptons will decay to final states including gauge bosons and charged leptons or neutrinos. Although there are no dedicated searches for these states at the LHC, generic searches for an anomalous production of multilepton final states [13] can be recast in order to set bounds on their mass and production cross-sections.

As mentioned above, one of the main goals of our paper is to determine the impact of the vector-like leptons on Higgs decays. Here, we put a special focus on the lepton flavour violating (LFV) Higgs decays $h \rightarrow e_{i} e_{j}$, that are a good example of LFV at colliders, a possibility less explored than LFV at low-energy experiments. Although several authors have been recently attracted by these non-standard Higgs decays [14-16], a fully-fledged analysis within a model with vector-like leptons is still lacking. We also discuss the possible effects of vector-like leptons on the $h \rightarrow \gamma \gamma$ and $Z \gamma$ decay rates. Especially the $h \rightarrow \gamma \gamma$ decay has received considerable attention in the recent literature (see e.g. [17-22]) due to the possibility of a significant enhancement of this mode. After the diphoton rate has evolved into a value compatible with the SM, the excitement about $h \rightarrow \gamma \gamma$ has clearly decreased. Nevertheless, it still remains as one of the places where NP might show up.

Although neutrino flavour oscillations constitute by themselves the proof that lepton flavour is not conserved, flavour violation in processes involving charged leptons has never been observed. This is well understood in the SM (minimally extended to include neutrino masses), where one expects tiny LFV rates. However, the situation changes dramatically when vector-like leptons are considered $[9,23]$. In the absence of flavour symmetries, the vector-like leptons will induce LFV processes, such as $e_{i} \rightarrow e_{j} \gamma$, with potentially large rates. Similarly, low-energy observables such as $g_{\mu}-2$ or the electron EDM are also affected by the presence of vector-like leptons $[24,25]$. We will show that many of these observables are clearly correlated. This will allow us to make definite predictions, which in turn will put this NP scenario under experimental test.

The paper is organized as follows: in section 2 we describe our setup. After a general review of the experimental situation, we study the collider phenomenology of vector-like leptons in section 3. In section 4, we present our results, with special emphasis on correlations among observables, and give the main predictions of this paper. Finally, we summarize our results and conclude in section 5 .

\section{The model}

In this paper we are interested in the class of models where the masses of SM charged leptons are generated via mixing with heavy vector-like leptons. The defining property of these fermions is that their two chiralities transform in the same way under the standard model gauge group, allowing for a Dirac mass term in the lagrangian. The particle content of model is shown in table 1 . We introduce 3 generations of chiral leptons, $l_{L}^{i}=\left(\nu_{L}^{i}, e_{L}^{i}\right)$, 


\begin{tabular}{|lccc|}
\hline & Generations & $\mathrm{SU}(2)_{L}$ & $\mathrm{U}(1)_{Y}$ \\
\hline$l_{L}=\left(\begin{array}{c}\nu_{L} \\
e_{L}\end{array}\right)$ & 3 & 2 & $-\frac{1}{2}$ \\
$e_{R}$ & 3 & 1 & -1 \\
\hline$L=\left(\begin{array}{c}N \\
E\end{array}\right)$ & 3 & 2 & $-\frac{1}{2}$ \\
$\tilde{E}$ & 3 & 1 & -1 \\
\hline
\end{tabular}

Table 1. Particle content of the model. The $l_{L}^{i}$ and $e_{R}^{i}$ states are the usual chiral leptons of the standard model, whereas the remaining states are new vector-like leptons. Our convention for the hypercharge follows $Q=T_{3 L}+Y$.

$e_{R}^{i}, i=1 \ldots 3$, and 3 generations of vector-like leptons $L^{i}=\left(N^{i}, E^{i}\right), \tilde{E}^{i}$, transforming as $2_{-1 / 2}$ and $1_{-1}$ under the electroweak gauge group. The vector-like leptons acquire masses from Dirac mass terms and from Yukawa couplings with the Higgs boson:

$$
\mathcal{L}_{F, c}=-M\left(\bar{L} C_{L} L+\overline{\tilde{E}} C_{R} \tilde{E}\right)-\left(\bar{L}_{L} Y \tilde{E}_{R} H+\bar{L}_{R} \tilde{Y} \tilde{E}_{L} H+\text { h.c. }\right) .
$$

Here $C_{L}, C_{R}, Y, \tilde{Y}$ are $3 \times 3$ matrices in the generation space, and we find it convenient to isolate a common scale $M$ from the vector-like mass terms. We can always rotate the basis such that $C_{L, R}$ are diagonal, however $Y$ and $\tilde{Y}$ are in general flavour non-diagonal and can induce flavour violating transitions. If $M \gg v$ and the elements of $C_{L, R}$ are $\mathcal{O}(1)$ then $M$ sets the mass scale of the heavy leptons. The chiral leptons do not acquire mass directly, but only via the mixing with the vector-like leptons:

$$
\mathcal{L}_{\text {mix }}=M\left(\bar{l}_{L} \lambda_{l} L_{R}+{\overline{E_{L}}}_{\lambda_{e}} e_{R}\right)+\text { h.c. }
$$

Here $\lambda_{i}$ are $3 \times 3$ matrices in the generation space.

This setup is inspired by composite Higgs models. In this framework, the chiral fermions correspond to the elementary states that mix with fermionic resonances in the composite sector. The Higgs field belongs to the composite sector and therefore has no direct couplings with the elementary fermions.

In the following, we will concentrate on the setup defined in eqs.(2.1) and (2.2). Therefore, any reference to the model should be understood as associated to this renormalizable lagrangian. Notice that our model does not contain higher dimensional operators. In more complete models, like those mentioned in the previous paragraph, there will be additional operators from the UV dynamics that will, in general, affect our results. We will comment on this possibility when necessary.

To understand the structure of the low-energy mass matrix of the charged leptons it is instructive to integrate out the vector-like fields $E$ and $\tilde{E}$. At the zero derivative level one obtains

$$
\mathcal{L}_{\mathrm{eff}}=-\frac{v}{\sqrt{2}} \bar{e}_{L} Y_{\mathrm{eff}} e_{R}+\text { h.c. } \quad Y_{\mathrm{eff}}=\lambda_{l} C_{L}^{-1}\left[Y+\frac{v^{2}}{2 M^{2}} Y C_{R}^{-1} \tilde{Y} C_{L}^{-1} Y+\ldots\right] C_{R}^{-1} \lambda_{e}
$$




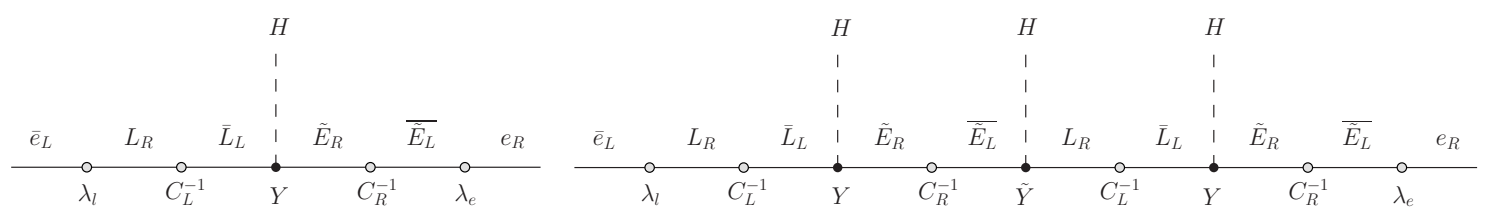

Figure 1. Diagramatic illustration of the effective Yukawa coupling.

This can be illustrated diagramatically, see figure 1. Clearly, the effective Yukawa couplings of the chiral leptons are proportional to the mixing parameters $\lambda_{l, e}$. By choosing hierarchical $\lambda$ 's it is possible to generate hierarchies of lepton masses and mixings, even if the Yukawa couplings are anarchic. Note that at the leading order the SM lepton masses depend on the Yukawa matrix $Y$ while the contribution of $\tilde{Y}$ is suppressed by $v^{2} / M^{2}$.

Similarly, working at the first derivative level one finds the Higgs couplings to leptons. These can be written as

$$
\mathcal{L}_{\text {eff }}=-\frac{h}{\sqrt{2}} \bar{e}_{L} c_{\text {eff }} e_{R}+\text { h.c. } \quad c_{\text {eff }}=Y_{\text {eff }}+\frac{v^{2}}{M^{2}} \lambda_{l} C_{L}^{-1} Y C_{R}^{-1} \tilde{Y} C_{L}^{-1} Y C_{R}^{-1} \lambda_{e},
$$

where we have neglected terms of order $\mathcal{O}\left(\lambda^{4}\right)$. The first term contains flavour diagonal Higgs couplings, similar to the ones in the SM. The second term is in general non-diagonal. Note that it vanishes when $\tilde{Y}=0$. Moreover, the flavour violating piece also contains a $v^{2} / M^{2}$ suppression.

We have not discussed neutrino masses so far. These can be accounted for by adding right-handed neutrinos (and their corresponding vector-like analogs) to our simple setup. Dirac and Majorana neutrino masses and be easily introduced in this way, see [8, 9, 11, 26]. Since extensions in this direction do not have any impact on charged lepton flavour violation, we choose not to discuss this issue any further.

\section{Current experimental situation and future projects}

In this section we review the current bounds and prospects of experimental searches for LFV and vector-like leptons.

\subsection{Bounds on lepton flavour violation}

The signatures of LFV processes are being searched for in multiple experiments. In table 2 we collect the present bounds and expected near-future sensitivity to the branching fraction of LFV lepton decays that may play a role in constraining models with vector-like leptons. Typically, the most stringent constraints on these models come from the limits on the $\mu \rightarrow e \gamma$ radiative decay. Recently, the MEG collaboration published a new limit, $\operatorname{Br}(\mu \rightarrow e \gamma)<5.7 \cdot 10^{-13}$, obtained from an analysis of the 2009-2011 data [27]. Future upgrades may reach the sensitivity of about $6 \cdot 10^{-14}$ after 3 years of acquisition time [28]. Limits on LFV radiative $\tau$ decays are less stringent, although they may be relevant in scenarios where LFV is Higgs mediated. The future generation of $B$ factories, in particular Belle II, will be able to shed new light on $\tau$ LFV decays [29, 30]. The 3-body decays of 


\begin{tabular}{|c|c|c|}
\hline LFV Process & Present Bound & Future Sensitivity \\
\hline$\mu \rightarrow e \gamma$ & $5.7 \times 10^{-13}[27]$ & $6 \cdot 10^{-14}[28]$ \\
$\tau \rightarrow e \gamma$ & $3.3 \times 10^{-8}[31]$ & $\sim 10^{-8}-10^{-9}[30]$ \\
$\tau \rightarrow \mu \gamma$ & $4.4 \times 10^{-8}[31]$ & $\sim 10^{-8}-10^{-9}[30]$ \\
$\mu \rightarrow 3 e$ & $1.0 \times 10^{-12}[32]$ & $\sim 10^{-16}[33]$ \\
$\tau \rightarrow 3 e$ & $2.7 \times 10^{-8}[34]$ & $\sim 10^{-9}-10^{-10}[30]$ \\
$\tau \rightarrow 3 \mu$ & $2.1 \times 10^{-8}[34]$ & $\sim 10^{-9}-10^{-10}[30]$ \\
$\mu^{-}, \mathrm{Au} \rightarrow e^{-}, \mathrm{Au}$ & $7.0 \times 10^{-13}[35]$ & \\
$\mu^{-}, \mathrm{Ti} \rightarrow e^{-}, \mathrm{Ti}$ & $4.3 \times 10^{-12}[36]$ & $\sim 10^{-18}[37]$ \\
\hline
\end{tabular}

Table 2. Current experimental bounds and future sensitivities for some low-energy LFV observables.

charged leptons, $e_{i} \rightarrow 3 e_{j}$, and the neutrinoless conversion in muonic atoms, can also be relevant in certain classes of models. However, these decays are suppressed compared to the radiative decays in models where the LFV amplitudes are dominated by dipole-type diagrams, as is the case in the model we study here.

The recent discovery of the Higgs boson opens the possibility of searching for LFV Higgs decays $h \rightarrow e_{i} e_{j}$, with $i \neq j$. First discussed in [38-41], these non-standard processes have gained some popularity recently. A significant branching fraction for these decays can be compatible with constraints from other LFV processes [14-16]. Although no official limits on the branching fractions exist at this moment, a recast of the $h \rightarrow \tau \tau$ search results allows one to place limits on $\operatorname{Br}(h \rightarrow \tau e / \mu)$ at the level of $10 \%$ [15]. As we shall see, the branching fractions predicted in our model are many orders of magnitude smaller, thus these limits currently play no role in constraining our model.

Finally, it is worthwhile to mention in this context the measurements of anomalous electric and magnetic dipole moments. Although these observables are lepton-flavour conserving, the relevant diagrams are typically generated along with the LFV ones. There is the long-standing $\sim 3.5 \sigma$ discrepancy between the predicted and measured anomalous muon magnetic moment, $\delta a_{\mu} \approx 2.9 \times 10^{-9}$ [42], which can be addressed in models with vector-like leptons. Currently, the experimental error on $a_{\mu}$ is at the level of $0.6 \times 10^{-9}$. The Muon g-2 experiment at Fermilab, which should start taking data in 2016, is expected to reduce that error by a factor of 3 . This will hopefully clarify whether the anomaly is due to new physics, although reducing the error on the theoretical prediction, currently of order $0.5 \times 10^{-9}$, may be more difficult. Another interesting observable is the electric dipole moment of the electron $d_{e}$. In this case the SM prediction is extremely small $d_{e}<10^{-38} e \mathrm{~cm}$ (see e.g. [43]), well below the current experimental sensitivity. On the other hand, models with $\mathrm{TeV}$ scale vector-like leptons whose couplings contain new $\mathrm{CP}$ violating phases may easily generate $d_{e}$ at an observable level. The most recent limit from the ACME collaboration obtained using the ThO molecules reads $d_{e}<8.9 \times 10^{-29} e \mathrm{~cm}$ [44]. This limit is expected to be improved by an order of magnitude in the near future. 


\subsection{Bounds on vector-like leptons}

The rate of LFV processes in our model strongly depends on the masses of vector-like leptons, and the latter can be directly constrained by collider searches [45]. The current bounds on vector-like leptons quoted by the PDG come from the LEP experiment [46]. Assuming a heavy charged lepton decaying to $W \nu$ with the $100 \%$ branching fraction, the lower bound on its mass is $100.8 \mathrm{GeV}$. Relaxing that assumption could lead to different limits, but in any case it would not be much larger than $\sim 100 \mathrm{GeV}$ [46]. To the best of our knowledge, no experimental bounds on vector-like leptons have been obtained so far by the LHC collaborations. However, it is possible to recast other existing searches. Ref. [9] used the ATLAS search for type-III seesaw mediators [47] to constrain composite leptons, with the resulting lower limit on the mass in the ballpark of $300 \mathrm{GeV}$. In the rest of this section, we discuss the constraints coming from the recent CMS multilepton search [13] that uses the full dataset at $\sqrt{s}=8 \mathrm{TeV}$.

We will focus on the situation in which the lowest lying heavy lepton state is significantly lighter than the others, and assume it is the only state produced with a non-negligible rate at the LHC. The lightest state decays to SM particles (leptons and weak gauge bosons) which may lead to multilepton final states when some of the gauge bosons decay leptonically. ${ }^{1}$ We consider the production of both the lightest charged lepton $E_{1}$ and the lightest neutral lepton $N_{1}$. For the charged one, the pair production cross section depends not only on the mass but also on the couplings to the $\mathrm{Z}$ boson, which in turn depend on the mixing angles in the charged lepton sector. Also the decay branching fractions depend on the mixing angles. Two limiting situations can be distinguished:

- Singlet, where the lightest state consists dominantly of a $\mathrm{SU}(2)_{L}$ singlet $\tilde{E}$ whose coupling to $Z$ is $g_{E_{1}}^{Z}=\frac{g_{Y}^{2}}{\sqrt{g_{L}^{2}+g_{Y}^{2}}}$. In this case, the only relevant process is the $E_{1}^{+} E_{1}^{-}$ pair production, while $N_{1}$ is assumed to be heavier and its production is neglected. In the singlet case, $E_{1}^{ \pm}$can decay to $Z l^{ \pm}$or to $W^{ \pm} \nu$. The ratio of these two partial widths is given by $2 \cos ^{2} \theta_{w}$, leading to $\operatorname{Br}\left(E_{1} \rightarrow Z l\right) \approx 0.4, \operatorname{Br}\left(E_{1} \rightarrow W \nu\right) \approx 0.6$.

- Doublet, where the lightest state consists dominantly of a $\mathrm{SU}(2)_{L}$ doublet $L$. It follows that $N_{1}$ and $E_{1}$ are approximately degenerate (we collectively denote them as $\left.L_{1}\right)$. The charged lepton couples to the $Z$ boson with strength $g_{E_{1}}^{Z}=-\frac{g_{L}^{2}-g_{Y}^{2}}{2 \sqrt{g_{L}^{2}+g_{Y}^{2}}}$, while the neutral one with $g_{N_{1}}^{Z}=\frac{\sqrt{g_{L}^{2}+g_{Y}^{2}}}{2}$. There is also the charged current interaction of the $E_{1}, N_{1}$ pair with strength $g_{E 1, N_{1}}^{W}=g_{L} / \sqrt{2}$. Thus, apart from the $E_{1}$ pair production, also the $N_{1} \bar{N}_{1}$ and $E_{1}^{\mp} N_{1}\left(\bar{N}_{1}\right)$ production processes should be taken into account. In the doublet case, $E_{1}$ decays to $Z l$ with $100 \%$ branching fraction, while $N_{1}$ decays to $W l$.

In this paper we present our results only for these limiting two cases. Furthermore, for simplicity we assume that $E_{1}$ and $N_{1}$ can decay to only one of the SM lepton flavours. To estimate the sensitivity of the CMS multilepton search we used Madgraph 5 [48] to

\footnotetext{
${ }^{1}$ For a related study on heavy lepton signatures at the LHC see [54].
} 

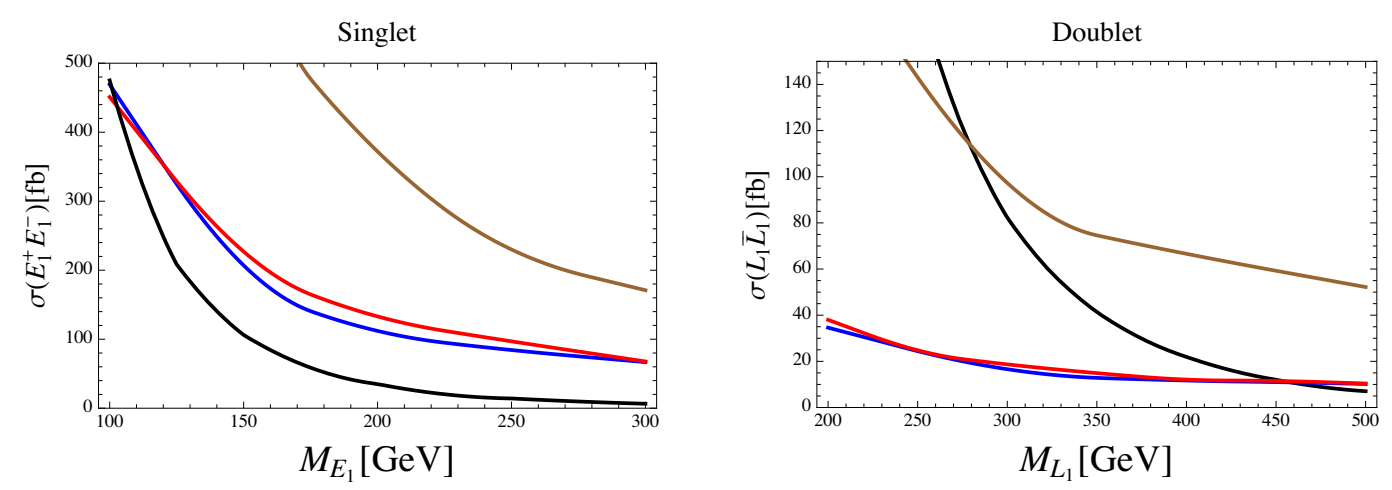

Figure 2. Upper limit on the pair production cross section at $\sqrt{s}=8 \mathrm{TeV}$ LHC of heavy leptons decaying to the electron (red), muon (blue), or tau (brown) SM flavour, for the singlet (left) and doublet (right) cases. The black line shows the production cross section of the heavy lepton via the Drell-Yan processes in our model.

simulate the $L_{1} \bar{L}_{1}$ production followed by decay $L_{1} \rightarrow V l$ where $V=W, Z$ and $l$ is a charged or neutral SM lepton. The events were then passed to Pythia [49] for showering and hadronization and to Delphes [50] for the CMS detector simulation. We repeated the CMS analysis cuts to determine the efficiency $\times$ acceptance for each of the multilepton categories defined in [13]. Then we defined the likelihood function for the SM background + heavy lepton signal (as a function of the heavy lepton production cross section) to be a simple product of Poisson likelihood for each category. In our procedure we ignored the theoretical errors on the SM background.

Our results are presented in figure 2. We give the $95 \%$ CL limits on the production cross section in the singlet and the doublet case, separately for heavy leptons decaying to electron, muon, and tau lepton flavour. To find the limit on the heavy lepton mass, this is compared to the production cross section due to the Drell-Yan processes in our model. In the singlet case the limits are weak: they are only slightly above $100 \mathrm{GeV}$ for decays to electrons and muons (thus marginally improving the LEP limits) and they are below $100 \mathrm{GeV}$ for decays to tau. In the doublet case the limits are more stringent, partly due to the larger production cross section, and partly to the larger efficiency. This leads to a lower limit on the mass of $L_{1}$ around $460 \mathrm{GeV}$ for the electron and muon decays, and around $280 \mathrm{GeV}$ for tau decays.

\section{Higgs decays and lepton flavour violation}

In this section we first derive approximate analytical expressions for the modification of Higgs decay branching ratios as well as for low-energy observables like charged lepton LFV decays and dipole moments, and finally we perform a numerical analysis.

\subsection{Higgs decays}

We denote the relative modification of Higgs decay widths compared to the SM as $R(h \rightarrow$ $X) \equiv \Gamma(h \rightarrow X) / \Gamma(h \rightarrow X)_{\mathrm{SM}}$. In our simple model with vector-like leptons only, this directly corresponds to the experimental signal strengths since corrections to the production 
cross section are negligible. We note however that in more complete models, e.g. composite models, the quark and gauge sectors can generally lead to sizable corrections to the production cross section.

Leptonic Higgs decays. The Higgs decays to electron, muon or $\tau$ pairs are corrected at tree level as

$$
R\left(h \rightarrow e_{i}^{+} e_{i}^{-}\right)=\left|\frac{c_{\mathrm{eff}}^{i i} v}{\sqrt{2} m_{e_{i}}}\right|^{2}=\left|1+\frac{\Delta c_{\mathrm{eff}}^{i i} v}{\sqrt{2} m_{e_{i}}}\right|^{2} .
$$

One can see from eqs. (2.3), (2.4) that this expression differs from 1 only for non-zero $\tilde{Y}^{i j}$. Interestingly, in this case also lepton flavour violating decays can be generated, which are forbidden in the SM. One can write

$$
\frac{\Gamma\left(h \rightarrow e_{i}^{ \pm} e_{j}^{\mp}\right)}{\Gamma\left(h \rightarrow e_{j}^{+} e_{j}^{-}\right)_{\mathrm{SM}}}=\frac{v^{2}}{2 m_{e_{j}}^{2}}\left(\left|c_{\mathrm{eff}}^{i j}\right|^{2}+\left|c_{\mathrm{eff}}^{j i}\right|^{2}\right)
$$

for $j>i$.

Higgs decays involving photons. We write the modification of the Higgs to diphoton decay rate as

$$
R(h \rightarrow \gamma \gamma)=\left|1+\frac{F_{H}^{\gamma \gamma}}{F_{\mathrm{SM}}^{\gamma \gamma}}\right|^{2}
$$

where

$$
F_{\mathrm{SM}}^{\gamma \gamma}=F_{1}\left(\frac{4 M_{W}^{2}}{m_{h}^{2}}\right)+\frac{4}{3} F_{1 / 2}\left(\frac{4 m_{t}^{2}}{m_{h}^{2}}\right) \approx-6.5
$$

Neglecting the mixing between the chiral and vector-like states, $F_{H}^{\gamma \gamma}$ is dominated by heavy fermion loops and can be written by means of the Higgs low energy theorem as [51]

$$
F_{H}^{\gamma \gamma} \approx \frac{4}{3} v \frac{\partial}{\partial v} \log \left(\operatorname{det} \mathcal{M}^{\dagger} \mathcal{M}\right) \approx-\frac{8}{3} \frac{v^{2}}{M^{2}} \operatorname{Tr}\left(C_{L}^{-1} Y C_{R}^{-1} \tilde{Y}\right)
$$

where

$$
\mathcal{M}=\left(\begin{array}{cc}
M C_{L} & \frac{v}{\sqrt{2}} Y \\
\frac{v}{\sqrt{2}} \tilde{Y} & M C_{R}
\end{array}\right)
$$

is the heavy block of the charge -1 fermion mass matrix. It can be seen from eq. (4.5) that $F_{H}$ can have either sign, so $h \rightarrow \gamma \gamma$ can be enhanced or suppressed.

For the $h \rightarrow Z \gamma$ decay, we focus for simplicity on the case of a single generation that has been worked out e.g. in [21]. One finds

$$
R(h \rightarrow Z \gamma)=\left|1+\frac{F_{H}^{Z \gamma}}{F_{\mathrm{SM}}^{Z \gamma}}\right|^{2},
$$


where $F_{\mathrm{SM}}^{Z \gamma} \approx 4.8$ and, neglecting the mixing between the chiral and vector-like fermions,

$$
F_{H}^{Z \gamma} \approx\left(1-4 \sin ^{2} \theta_{w}\right) \frac{1}{3} \frac{v^{2}}{M^{2}} Y \tilde{Y}+O\left(\frac{\left(C_{L}-C_{E}\right)^{2}}{\left(C_{L}+C_{E}\right)^{2}}\right) .
$$

Compared to $h \rightarrow \gamma \gamma$, this contribution is accidentally suppressed by $1-4 \sin ^{2} \theta_{w} \approx 0.08$. In the three-generation case, the correlation between $h \rightarrow \gamma \gamma$ and $h \rightarrow Z \gamma$ depends additionally on $O(1)$ factors that depend on the flavour structure of the $Y$ and $\tilde{Y}$, so we do not expect a clear-cut correlation.

\subsection{Low-energy observables}

Radiative lepton decay. Normalizing the branching ratio of the radiative LFV decay to the semileptonic decay, one has

$$
\frac{\operatorname{BR}\left(e_{i} \rightarrow e_{j} \gamma\right)}{\operatorname{BR}\left(e_{i} \rightarrow e_{j} \nu_{i} \bar{\nu}_{j}\right)}=\frac{3 \alpha}{4 \pi G_{F}^{2}}\left(\left|A_{L}^{i j}\right|^{2}+\left|A_{R}^{i j}\right|^{2}\right)
$$

where

$$
\begin{aligned}
& A_{L}^{i j}=\frac{v^{2}}{2 M^{2}} \frac{1}{\sqrt{2} m_{e_{i}} v}\left(\lambda_{l} C_{L}^{-1} Y C_{R}^{-1} \tilde{Y} C_{L}^{-1} Y C_{R}^{-1} \lambda_{e}\right)_{i j}, \\
& A_{R}^{i j}=\frac{v^{2}}{2 M^{2}} \frac{1}{\sqrt{2} m_{e_{i}} v}\left(\lambda_{l} C_{L}^{-1} Y C_{R}^{-1} \tilde{Y} C_{L}^{-1} Y C_{R}^{-1} \lambda_{e}\right)_{j i} .
\end{aligned}
$$

By comparison to (2.4), one sees that these expressions are proportional to the effective flavour-violating Higgs couplings and one can write

$$
A_{L}^{i j}=\frac{c_{\mathrm{eff}}^{i j}}{2 \sqrt{2} m_{e_{i}} v}, \quad \quad A_{R}^{i j}=\frac{c_{\mathrm{eff}}^{j i}}{2 \sqrt{2} m_{e_{i}} v} .
$$

Eqs. (4.2), (4.12) leads to a perfect correlation between the LFV Higgs decays $h \rightarrow e_{i} e_{j}$ and the radiative $e_{i} \rightarrow e_{j} \gamma$ decays,

$$
\frac{\mathrm{BR}\left(h \rightarrow e_{i} e_{j}\right)}{\operatorname{BR}\left(e_{i} \rightarrow e_{j} \gamma\right)}=\frac{\mathrm{BR}\left(h \rightarrow e_{i} e_{i}\right)_{\mathrm{SM}}}{\operatorname{BR}\left(e_{i} \rightarrow e_{j} \nu_{i} \bar{\nu}_{j}\right)} \frac{4 \pi}{3 \alpha}
$$

Inserting numbers, one obtains

$$
\begin{gathered}
\mathrm{BR}(h \rightarrow \tau \mu)<8.6 \times 10^{-6}\left[\frac{\mathrm{BR}(\tau \rightarrow \mu \gamma)}{4.4 \times 10^{-8}}\right], \\
\mathrm{BR}(h \rightarrow \tau e)<6.2 \times 10^{-6}\left[\frac{\mathrm{BR}(\tau \rightarrow e \gamma)}{3.3 \times 10^{-8}}\right], \\
\mathrm{BR}(h \rightarrow \mu e)<6.7 \times 10^{-14}\left[\frac{\mathrm{BR}(\mu \rightarrow e \gamma)}{5.7 \times 10^{-13}}\right],
\end{gathered}
$$

where the branching ratios in square brackets are normalized to the current upper bounds. We conclude that LFV Higgs decay branching ratios are at least four orders of magnitude smaller than the Higgs decays to tau pairs and are thus most probably unobservable. 
Muon anomalous magnetic moment. In the flavour conserving case, the dipole operators contribute to the anomalous magnetic moment of the muon, $a_{\mu}=\left(g_{\mu}-2\right) / 2$ [24]. One can again write it in terms of $c_{\text {eff }}$ as

$$
\delta a_{\mu} \approx-\frac{m_{\mu}}{8 \sqrt{2} \pi^{2} v} \operatorname{Re} \Delta c_{\mathrm{eff}}^{\mu \mu} .
$$

Together with eq. (4.1), one can then write for small $\Delta c_{\mathrm{eff}}^{\mu \mu}$

$$
R\left(h \rightarrow \mu^{+} \mu^{-}\right) \approx 1-\left[\frac{\delta a_{\mu}}{1.6 \times 10^{-9}}\right] .
$$

If the current discrepancy is due to NP, in this model one would thus expect a significant suppression of the $h \rightarrow \mu \mu$ branching ratio.

Electron electric dipole moment. The electron EDM is given by

$$
\frac{d_{e}}{e} \approx-\frac{1}{16 \sqrt{2} \pi^{2} v} \operatorname{Im} \Delta c_{\mathrm{eff}}^{e e}
$$

Assuming the flavour structure of the $Y, \tilde{Y}$ and $C_{L, R}$ do not lead to any additional suppression, one expects roughly

$$
\left|\Delta c_{\mathrm{eff}}^{e e}\right| \sim \frac{m_{e}}{m_{\mu}}\left|\Delta c_{\mathrm{eff}}^{\mu \mu}\right|
$$

and thus obtains a correlation between $d_{e}$ and $g_{\mu}-2$, namely

$$
\begin{aligned}
d_{e} & \sim \frac{e}{2} \frac{m_{e}}{m_{\mu}} \frac{\delta a_{\mu}}{m_{\mu}} \sin \varphi_{e} \\
& \approx 5 \times 10^{-25} \mathrm{ecm} \times\left[\frac{\delta a_{\mu}}{10^{-9}}\right] \sin \varphi_{e}
\end{aligned}
$$

where $\varphi_{e}$ is the phase of $\Delta c_{\text {eff }}^{e e}$ Consequently, an explanation of the muon $g-2$ anomaly would require a phase below $10^{-4}$ to be in agreement with the recent bound from the ACME experiment $d_{e} \leq 8.7 \times 10^{-29} e \mathrm{~cm}$. For an $O(1)$ phase, one would instead require $M \gtrsim$ $23 \sqrt{|Y \tilde{Y}|} \mathrm{TeV}$ to fulfill the EDM bound and the contribution to $g_{\mu}-2$ would be negligible.

Before moving on to the numerical analysis, we briefly comment on the dependence of the phenomenology on the choice of fermion representations. Although we have focused on the minimal case of one $\mathrm{SU}(2)_{L}$ doublet and one singlet mixing with the SM leptons, also more complicated possibilities like triplets are conceivable, or bi-doublets under a custodial group $\mathrm{SU}(2)_{L} \times \mathrm{SU}(2)_{R}$ as in many composite models. In that case, a different $O(1)$ factor can appear in front of $\Delta c_{\text {eff }}$, i.e. the modification of the Higgs coupling with respect to the SM. In addition, a different $O(1)$ factor can multiply all the amplitudes induced by the dipole operator. ${ }^{2}$ However, the qualitative correlations between the tree level Higgs decays and the dipole-induced observables will still hold. The most significant change occurs in the loop-induced $h \rightarrow \gamma \gamma$ and $h \rightarrow Z \gamma$ decays in the presence of large representations with high charges [21].

\footnotetext{
${ }^{2}$ These factors have been computed for several different choices of representations in ref. [24].
} 


\subsection{Numerical analysis}

Having discussed the most important indirect probes of our scenario, we proceed with a numerical analysis, taking into account the direct bounds as discussed in section 3.2. To this end, we conduct a scan over the model parameters in the following ranges,

$$
\begin{array}{rlrl}
\left(M C_{L, R}\right)_{i i} & \in[100,1500] \mathrm{GeV}, & \frac{\left(\lambda_{l, e}\right)_{i i}}{\left(M C_{L, R}\right)_{i i}} & \in\left[10^{-5}, 1\right], \\
\left|Y_{i i}\right|,\left|\tilde{Y}_{i i}\right| & \in[1 / 3,3], & \left|Y_{i j}\right|,\left|\tilde{Y}_{i j}\right| \in\left[10^{-5}, 1\right],
\end{array}
$$

where we have scanned the masses and diagonal elements of the Yukawa matrices linearly and the mixings $\lambda_{l, e}$ and the off-diagonal elements of the Yukawa matrices logarithmically, since they can span many orders of magnitude. We have also scanned the phases of the $Y$ and $\tilde{Y}$ between 0 and $2 \pi$.

After imposing that the three charged lepton masses be reproduced correctly, which fixes three of the six parameters in $\lambda_{l, e}$, we remove all points where one of the heavy mass eigenstates violates the direct LEP bound of $100 \mathrm{GeV}$. We also impose the LEP bounds on modified $Z$ couplings to charged lepton pairs [52] that constrain the relative modification to the per mille level. This constraint is particularly relevant for the $\tau$, that has the largest mixing with the vector-like leptons. For the points surviving these constraints, we compute the cross section times branching ratio for the pair production and decay to $W$ or $Z$ and leptons and impose the LHC bounds as discussed in section 3.2. Points that are in violation of this bound are shown in light gray in the following plots, while allowed points are shown in blue.

Finally, we compute all the observables discussed in sections 4.1 and 4.2. We stress that we do not employ the approximate analytical formulae, which have been presented above only to get an analytical understanding of the correlations. Rather, we diagonalize the full $9 \times 9$ mass matrix for charge -1 fermion mass eigenstates (and correspondingly for the neutral mass eigenstates) and also compute the loop-induced observables taking into account the full dependence of the loop functions without any approximations.

Figure 3 shows the results for the correlations between flavour violating Higgs decays and radiative lepton decays. The green lines show the approximate result given in eqs. (4.14) and (4.15) which can be seen to be fulfilled to an excellent precision, in particular once the LHC direct constraints are taken into account. The conclusion that flavourviolating Higgs decays will be unobservable at the LHC in this model is thus robust. ${ }^{3}$

The left-hand panel of figure 4 shows the correlation of the Higgs to diphoton decay with the muon anomalous magnetic moment. Although there is now clear correlation in the three-generation flavour-anarchic case we consider, we observe that a solution of the magnetic muon anomaly typically leads to a suppression of $h \rightarrow \gamma \gamma$. The right-hand panel shows the correlation between $h \rightarrow \gamma \gamma$ and $h \rightarrow Z \gamma$. As discussed above, the effects in the latter decay are typically smaller due to the accidental suppression by $\left(1-4 \sin \theta_{w}^{2}\right)$.

\footnotetext{
${ }^{3}$ This conclusion applies to the model explicitly defined in eqs. (2.1), (2.2) and, provided they do not have additional sources of LFV, to all models that reduce to that renormalizable lagrangian at low energies. Notice, however, that other new physics at high energies may generate non-dipole LFV operators contributing to $h \rightarrow e_{i} e_{j}$ and lead to observable flavour violating Higgs decays.
} 

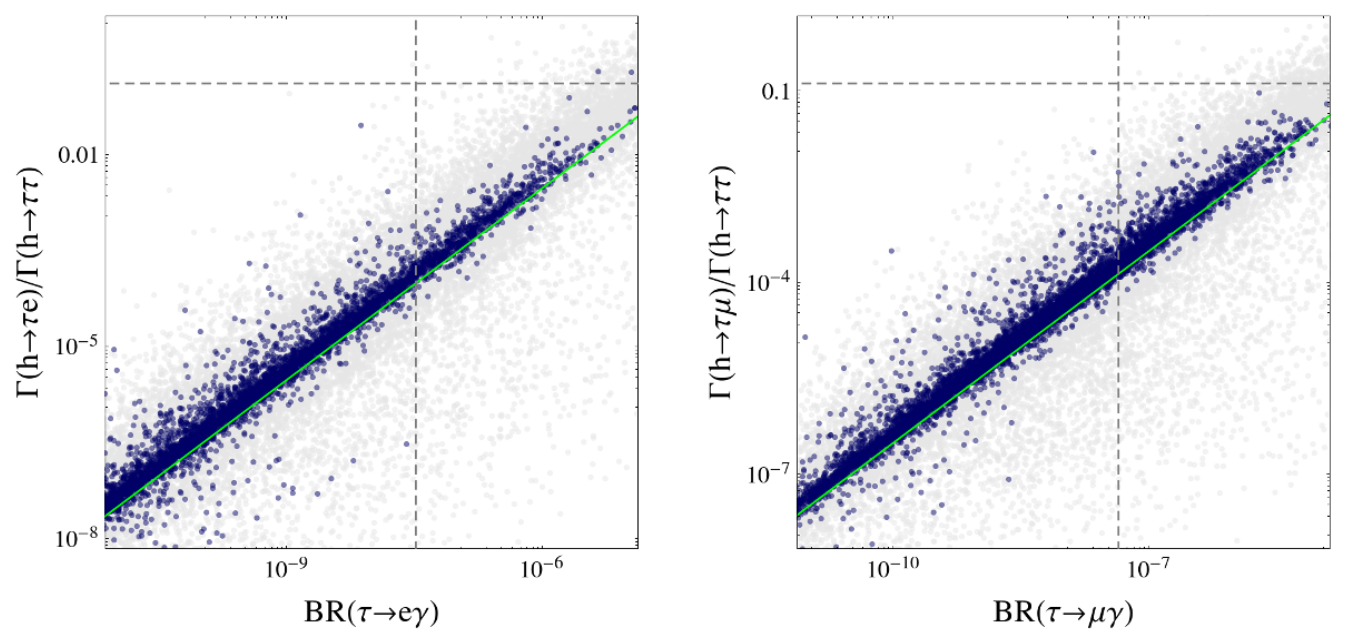

Figure 3. Correlation between flavour-violating Higgs decays and radiative lepton decays. Gray points are ruled out by LHC direct searches. The experimentally allowed region is left of and below the dashed lines. The green lines correspond to eqs. (4.15) and (4.14).
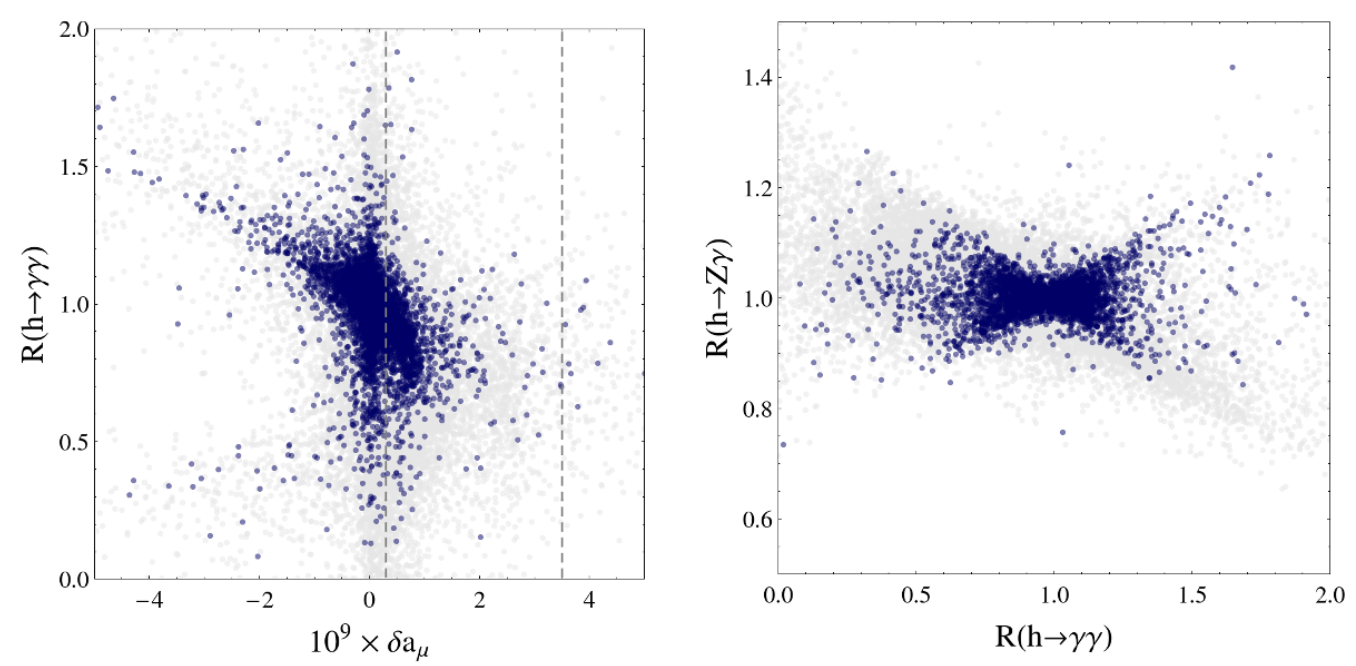

Figure 4. Correlation between $h \rightarrow \gamma \gamma, h \rightarrow Z \gamma$ and the muon anomalous magnetic moment. Gray points are ruled out by LHC direct searches. The dashed lines show the $2 \sigma$ experimental region.

Figure 5 shows the correlation between the muon anomalous magnetic moment and the $h \rightarrow \mu \mu$ decay as well as the electron EDM. In the first case, we also show the approximate relation given in eq. (4.18) as a solid green line. We can see that this relation is fulfilled to a good precision once the LHC direct constraints are taken into account, but there are also some viable points with sizable positive contribution to $\delta a_{\mu}$, as preferred by experiment, but no significant modification of the $h \rightarrow \mu \mu$ decay rate. The second plot shows that, as expected from the above discussion, allowing the phases in the Yukawas to be of $O(1)$, 

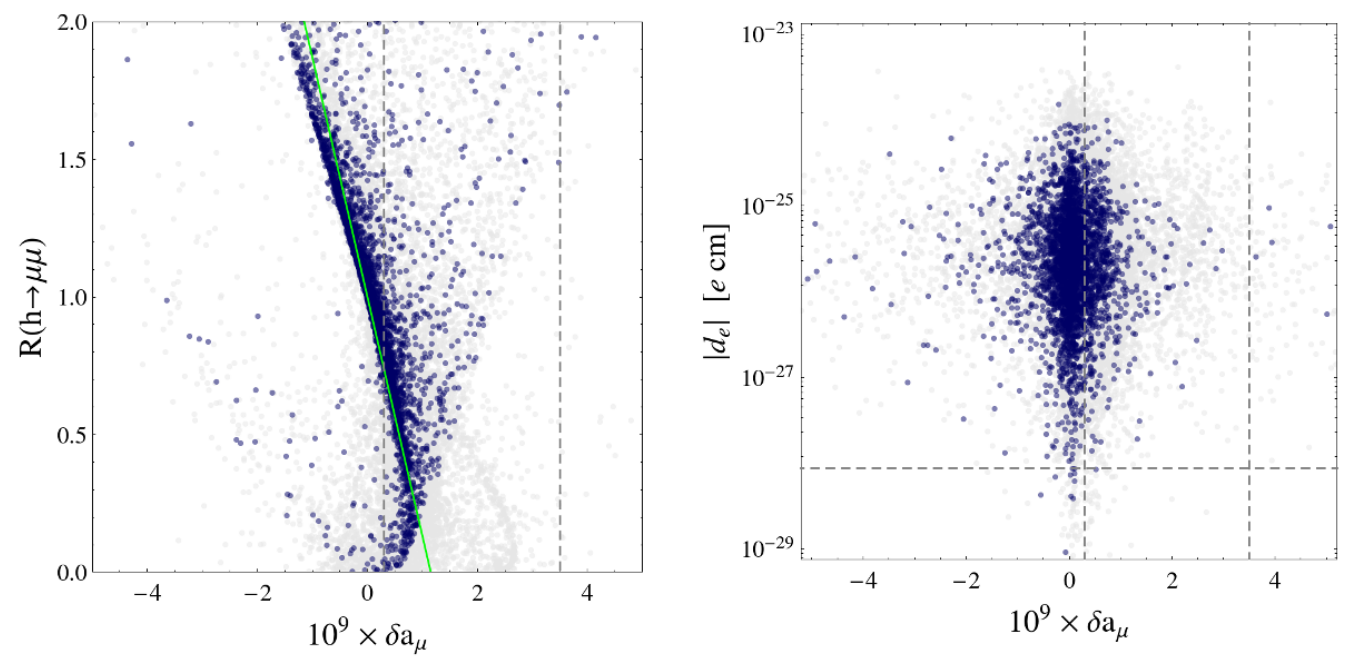

Figure 5. Correlation of the muon anomalous magnetic moment with $h \rightarrow \mu \mu$ and the electron EDM, respectively. Gray points are ruled out by LHC direct searches. The dashed lines show the $2 \sigma$ experimental region for $\delta a_{\mu}$ and the $90 \%$ C.L. upper bound on $\left|d_{e}\right|$. The green line corresponds to eq. (4.18).

one typically produces a too large electron EDM that is ruled out by the bound from the ACME collaboration.

\section{$5 \quad$ Summary and conclusions}

We have explored the phenomenology of a simple model with vector-like leptons, with detailed studies of their impact on lepton flavour violation, Higgs decays and collider physics.

After the discovery of the Higgs boson, the next step is to determine its properties and look for non-standard signatures. In this paper we have explored how the presence of vector-like leptons might affect two Higgs decays channels: (i) $h \rightarrow e_{i} e_{j}$, with $i \neq j$, and (ii) $h \rightarrow \gamma \gamma$. In the model under consideration (defined by eqs. (2.1), (2.2)), our results clearly show that the strong upper bounds on $\mathrm{BR}\left(e_{i} \rightarrow e_{j} \gamma\right)$ preclude any possibility to observe Higgs LFV decays at the LHC. Therefore, in case these were observed at the LHC, vectorlike leptons would be ruled out as the only source of LFV and additional contributions to non-dipole LFV operators would be necessary. On the other hand, the Higgs diphoton rate can actually be modified due to the new contributions induced by the vector-like leptons. These do not have a definite sign and thus $\mathrm{BR}(h \rightarrow \gamma \gamma)$ can be either enhanced or suppressed with respect to the SM prediction. Effects in $\mathrm{BR}(h \rightarrow Z \gamma)$ are typically smaller.

Vector-like leptons also contribute to the dipole operators involved in $g_{\mu}-2$. These contributions are found to be correlated with the corrections to $\mathrm{BR}(h \rightarrow \mu \mu)$. The conclusion is that, if the discrepancy in $g_{\mu}-2$ is explained thanks to the vector-like leptons, we expect a significant suppression of the $h \rightarrow \mu \mu$ branching ratio. At the same time, a large enough contribution to $g_{\mu}-2$ requires generic CP violating phases in the Yukawa matrices to be of order $\mathcal{O}\left(10^{-4}\right)$, otherwise the bound on the electron EDM would be violated. 
In what concerns high-energy signatures of vector-like leptons, we have focused on their pair production at the LHC. Using the recent multilepton search released by the CMS collaboration, we have derived $95 \%$ C.L. limits on the mass of the vector-like lepton as a function of its production cross-section. For a charged vector-like lepton that is a singlet of the SM SU $(2)_{L}$ group, the CMS search does not improve on the previous LEP limits of $m_{E} \gtrsim 100 \mathrm{GeV}$. In contrast, for a pair of charged and neutral vector-like leptons that form a $\mathrm{SU}(2)_{L}$ doublet the CMS limits turn out to be non-trivial. The limits for a heavy lepton doublet decaying to the $e$ or $\mu$ flavours are $m_{L} \gtrsim 450 \mathrm{GeV}$. In the case of decays to the $\tau$ flavour the limits are less stringent: $m_{L} \gtrsim 270 \mathrm{GeV}$.

Vector-like leptons appear in many well-motivated extensions of the SM, like composite Higgs models, or models with warped extra dimensions and the Lagrangian defined by eqs. (2.1), (2.2) can be seen as the low-energy limit of some of these theories. An interesting question is how the correlations we identified would be modified by the additional UV dynamics in these models. Concerning the upper bounds on flavour-violating Higgs decays we found, see eqs. (4.14)-(4.16), we note that the dipole operators contributing to radiative LFV decays arising at the loop level in our model may receive additional contributions from higher-dimensional operators in more complete models, but barring accidental cancellations, such contributions would only strengthen the bounds. We thus expect that our conclusion that LFV Higgs decays are unobservable is in fact valid in a much larger class of models that have our model as low-energy limit. A complete study of this interesting question is beyond the scope of our present study.

Note added. While finalizing the manuscript, we became aware of a talk by M. Beneke [53] where the connection between LFV Higgs decays and dipole transitions was discussed in the context of Randall-Sundrum models.

\section{Acknowledgments}

We thank Michele Redi and Paride Paradisi for useful discussions. D.M.S. was supported by the Advanced Grant EFT4LHC of the European Research Council (ERC), the Cluster of Excellence Precision Physics, Fundamental Interactions and Structure of Matter (PRISMA - EXC 1098) as well as the Cluster of Excellence Origin and Structure of the Universe and thanks the Galileo Galilei Institute for Theoretical Physics for the hospitality and the INFN for partial support while some of this work was carried out. A.V. acknowledges partial support from the ANR project CPV-LFV-LHC NT09-508531. The work of A.F. was supported by the ERC advanced grant Higgs@LHC.

Open Access. This article is distributed under the terms of the Creative Commons Attribution License (CC-BY 4.0), which permits any use, distribution and reproduction in any medium, provided the original author(s) and source are credited. 


\section{References}

[1] ATLAS collaboration, Observation of a new particle in the search for the Standard Model Higgs boson with the ATLAS detector at the LHC, Phys. Lett. B 716 (2012) 1 [arXiv:1207.7214] [INSPIRE].

[2] CMS collaboration, Observation of a new boson at a mass of $125 \mathrm{GeV}$ with the CMS experiment at the LHC, Phys. Lett. B 716 (2012) 30 [arXiv:1207.7235] [INSPIRE].

[3] A. Falkowski, F. Riva and A. Urbano, Higgs at last, JHEP 11 (2013) 111 [arXiv:1303.1812] [INSPIRE].

[4] P.P. Giardino, K. Kannike, I. Masina, M. Raidal and A. Strumia, The universal Higgs fit, arXiv: 1303.3570 [INSPIRE].

[5] J. Ellis and T. You, Updated global analysis of Higgs couplings, JHEP 06 (2013) 103 [arXiv: 1303.3879] [INSPIRE].

[6] A. Djouadi and G. Moreau, The couplings of the Higgs boson and its CP properties from fits of the signal strengths and their ratios at the $7+8 \mathrm{TeV} L H C$, arXiv: 1303.6591 [INSPIRE].

[7] K. Fujikawa, A vector-like extension of the Standard Model, Prog. Theor. Phys. 92 (1994) 1149 [hep-ph/9411258] [INSPIRE].

[8] B. Keren-Zur et al., On partial compositeness and the CP asymmetry in charm decays, Nucl. Phys. B 867 (2013) 394 [arXiv:1205.5803] [InSPIRE].

[9] M. Redi, Leptons in composite MFV, JHEP 09 (2013) 060 [arXiv: 1306.1525] [INSPIRE].

[10] R. Contino, T. Kramer, M. Son and R. Sundrum, Warped/composite phenomenology simplified, JHEP 05 (2007) 074 [hep-ph/0612180] [INSPIRE].

[11] K. Agashe, T. Okui and R. Sundrum, A common origin for neutrino anarchy and charged hierarchies, Phys. Rev. Lett. 102 (2009) 101801 [arXiv:0810.1277] [INSPIRE].

[12] P.H. Frampton, P.Q. Hung and M. Sher, Quarks and leptons beyond the third generation, Phys. Rept. 330 (2000) 263 [hep-ph/9903387] [inSPIRE].

[13] CMS collaboration, A search for anomalous production of events with three or more leptons using $19.5 \mathrm{fb}$ of $\sqrt{s}=8 \mathrm{TeV}$ LHC data, CMS-PAS-SUS-13-002, CERN, Geneva Switzerland (2013).

[14] G. Blankenburg, J. Ellis and G. Isidori, Flavour-changing decays of a 125 GeV Higgs-like particle, Phys. Lett. B 712 (2012) 386 [arXiv:1202.5704] [InSPIRE].

[15] R. Harnik, J. Kopp and J. Zupan, Flavor violating Higgs decays, JHEP 03 (2013) 026 [arXiv: 1209.1397] [INSPIRE].

[16] S. Davidson and P. Verdier, LHC sensitivity to the decay $h \rightarrow \tau^{ \pm} m u^{\mp}$, Phys. Rev. D 86 (2012) 111701 [arXiv:1211.1248] [INSPIRE].

[17] N. Arkani-Hamed, K. Blum, R.T. D'Agnolo and J. Fan, $2: 1$ for naturalness at the LHC?, JHEP 01 (2013) 149 [arXiv:1207.4482] [INSPIRE].

[18] J. Kearney, A. Pierce and N. Weiner, Vectorlike fermions and Higgs couplings, Phys. Rev. D 86 (2012) 113005 [arXiv: 1207.7062] [INSPIRE].

[19] L.G. Almeida, E. Bertuzzo, P.A.N. Machado and R.Z. Funchal, Does $H \rightarrow \gamma \gamma$ taste like vanilla new physics?, JHEP 11 (2012) 085 [arXiv:1207.5254] [INSPIRE]. 
[20] A. Carmona and F. Goertz, Custodial leptons and Higgs decays, JHEP 04 (2013) 163 [arXiv:1301.5856] [INSPIRE].

[21] W. Altmannshofer, M. Bauer and M. Carena, Exotic leptons: Higgs, flavor and collider phenomenology, JHEP 01 (2014) 060 [arXiv:1308.1987] [INSPIRE].

[22] K. Ishiwata and M.B. Wise, Higgs properties and fourth generation leptons, Phys. Rev. D 84 (2011) 055025 [arXiv: 1107.1490] [InSPIRE].

[23] K. Ishiwata and M.B. Wise, Phenomenology of heavy vectorlike leptons, Phys. Rev. D 88 (2013) 055009 [arXiv: 1307.1112] [INSPIRE].

[24] K. Kannike, M. Raidal, D.M. Straub and A. Strumia, Anthropic solution to the magnetic muon anomaly: the charged see-saw, JHEP 02 (2012) 106 [Erratum ibid. 10 (2012) 136] [arXiv:1111.2551] [INSPIRE].

[25] R. Dermisek and A. Raval, Explanation of the muon g-2 anomaly with vectorlike leptons and its implications for Higgs decays, Phys. Rev. D 88 (2013) 013017 [arXiv:1305.3522] [INSPIRE].

[26] A.M. Iyer and S.K. Vempati, Lepton masses and flavor violation in Randall Sundrum model, Phys. Rev. D 86 (2012) 056005 [arXiv: 1206.4383] [InSPIRE].

[27] MEG collaboration, J. Adam et al., New constraint on the existence of the $\mu^{+} \rightarrow e^{+} \gamma$ decay, Phys. Rev. Lett. 110 (2013) 201801 [arXiv:1303.0754] [inSPIRE].

[28] A.M. Baldini et al., MEG upgrade proposal, arXiv:1301.7225 [INSPIRE].

[29] SuperB collaboration, B. O'Leary et al., SuperB progress reports - physics, arXiv: 1008.1541 [INSPIRE].

[30] Belle and Belle II collaborations, K. Hayasaka, Results and prospects on lepton flavor violation at Belle/Belle II, J. Phys. Conf. Ser. 408 (2013) 012069 [INSPIRE].

[31] BABAR collaboration, B. Aubert et al., Searches for lepton flavor violation in the decays $\tau^{ \pm} \rightarrow e^{ \pm} \gamma$ and $\tau^{ \pm} \rightarrow \mu^{ \pm} \gamma$, Phys. Rev. Lett. 104 (2010) 021802 [arXiv:0908.2381] [INSPIRE].

[32] SINDRUM collaboration, U. Bellgardt et al., Search for the decay $\mu^{+} \rightarrow e^{+} e^{+} e^{-}$, Nucl. Phys. B 299 (1988) 1 [INSPIRE].

[33] A. Blondel et al., Research proposal for an experiment to search for the decay $\mu \rightarrow$ eee, arXiv:1301.6113 [INSPIRE].

[34] K. Hayasaka et al., Search for lepton flavor violating $\tau$ decays into three leptons with 719 million produced $\tau^{+} \tau^{-}$pairs, Phys. Lett. B 687 (2010) 139 [arXiv:1001.3221] [INSPIRE].

[35] SINDRUM II collaboration, W.H. Bertl et al., A search for muon to electron conversion in muonic gold, Eur. Phys. J. C 47 (2006) 337 [INSPIRE].

[36] SINDRUM II collaboration, C. Dohmen et al., Test of lepton flavor conservation in $\mu \rightarrow e$ conversion on titanium, Phys. Lett. B 317 (1993) 631 [INSPIRE].

[37] PRIME WORKIng Group collaboration, Search for the $\mu \rightarrow e$ conversion process at an ultimate sensitivity of the order of $10^{-18}$ with prism, unpublished, LOI to J-PARC 50-GeV PS, LOI-25, http://www-ps.kek.jp/jhf-np/LOIlist/pdf/L25.pdf, Japan.

[38] A. Pilaftsis, Lepton flavor nonconservation in Ho decays, Phys. Lett. B 285 (1992) 68 [INSPIRE]. 
[39] J.L. Diaz-Cruz and J.J. Toscano, Lepton flavor violating decays of Higgs bosons beyond the Standard Model, Phys. Rev. D 62 (2000) 116005 [hep-ph/9910233] [INSPIRE].

[40] T. Han and D. Marfatia, $h \rightarrow \mu \tau$ at hadron colliders, Phys. Rev. Lett. 86 (2001) 1442 [hep-ph/0008141] [INSPIRE].

[41] K.A. Assamagan, A. Deandrea and P.-A. Delsart, Search for the lepton flavor violating decay $A^{0} / H^{0} \rightarrow \tau^{ \pm} \mu^{\mp}$ at hadron colliders, Phys. Rev. D 67 (2003) 035001 [hep-ph/0207302] [INSPIRE].

[42] M. Davier, A. Hoecker, B. Malaescu and Z. Zhang, Reevaluation of the hadronic contributions to the muon g-2 and to $\alpha\left(M_{Z}^{2}\right)$, Eur. Phys. J. C 71 (2011) 1515 [Erratum ibid. C 72 (2012) 1874] [arXiv:1010.4180] [InSPIRE].

[43] M. Pospelov and A. Ritz, Electric dipole moments as probes of new physics, Annals Phys. 318 (2005) 119 [hep-ph/0504231] [INSPIRE].

[44] ACME collaboration, J. Baron et al., Order of magnitude smaller limit on the electric dipole moment of the electron, Science 343 (2014) 269 [arXiv:1310.7534] [INSPIRE].

[45] S.D. Thomas and J.D. Wells, Phenomenology of massive vectorlike doublet leptons, Phys. Rev. Lett. 81 (1998) 34 [hep-ph/9804359] [INSPIRE].

[46] Particle Data Group collaboration, J. Beringer et al., Review of particle physics (RPP), Phys. Rev. D 86 (2012) 010001 [INSPIRE].

[47] ATLAS collaboration, Search for type III seesaw model heavy fermions in events with four charged leptons using $5.8 \mathrm{fb}^{-1}$ of $\sqrt{s}=8 \mathrm{TeV}$ data with the ATLAS detector, ATLAS-CONF-2013-019, CERN, Geneva Switzerland (2013).

[48] J. Alwall, M. Herquet, F. Maltoni, O. Mattelaer and T. Stelzer, MadGraph 5: going beyond, JHEP 06 (2011) 128 [arXiv:1106.0522] [INSPIRE].

[49] T. Sjöstrand, S. Mrenna and P.Z. Skands, PYTHIA 6.4 physics and manual, JHEP 05 (2006) 026 [hep-ph/0603175] [INSPIRE].

[50] DELPHES 3 collaboration, J. de Favereau et al., DELPHES 3, a modular framework for fast simulation of a generic collider experiment, JHEP 02 (2014) 057 [arXiv:1307.6346] [INSPIRE].

[51] M. Carena, I. Low and C.E.M. Wagner, Implications of a modified Higgs to diphoton decay width, JHEP 08 (2012) 060 [arXiv:1206.1082] [INSPIRE].

[52] AlePh, DelPhi, L3, OPAL, SLD, LeP Electroweak Working Group, SLD Electroweak Group and SLD Heavy Flavour Group collaborations, S. Schael et al., Precision electroweak measurements on the $Z$ resonance, Phys. Rept. 427 (2006) 257 [hep-ex/0509008] [INSPIRE].

[53] M. Beneke, Flavour physics, talk given at the Latsis Symposium, Zurich Switzerland 6 June 2013.

[54] J.A. Aguilar-Saavedra, Heavy lepton pair production at LHC: model discrimination with multi-lepton signals, Nucl. Phys. B 828 (2010) 289 [arXiv:0905.2221] [INSPIRE]. 